\title{
Desenvolvimento e validação de instrumento de avaliação dos aspectos que influenciam a solicitação de exames*
}

\author{
Development and validation of an instrument to evaluate \\ the aspects that influence exam request
}

\author{
Maria Fernanda Santos Figueiredo Brito ${ }^{1,2}$, Luciana Mendes Araújo Borém¹, \\ Romerson Brito Messias ${ }^{1,2}$, Marise Fagundes Silveira', \\ Ana Maria Vitricia de Souza ${ }^{1}$, Maisa Tavares de Souza Leite ${ }^{3}$, \\ João Felício Rodrigues Neto ${ }^{1}$
}

\begin{abstract}
Resumo
Introdução: Os exames integram o sistema de saúde e contribuem para o avanço da assistência. A sua solicitação pelos médicos deve ser realizada adequadamente, podendo ser influenciada por diferentes aspectos. Objetivo: Desenvolver e validar um instrumento de avaliação de aspectos que influenciam a solicitação de exames. Método: Trata-se de um estudo transversal e analítico que passou pelas seguintes etapas: 1) identificação dos aspectos que influenciam a solicitação de exames na prática médica; 2) verificação da validade aparente e validade de conteúdo; 3) verificação da validade de construto; 4) análise da confiabilidade; 5) verificação da validade discriminante, aplicada a 158 médicos de família e emergencistas no norte de Minas Gerais. Resultados: $O$ instrumento permaneceu com 36 itens, psicometricamente válidos, agrupados em cinco dimensões: particularidade pessoal do médico; estrutura e processos do sistema de saúde; características do paciente; preparo profissional; e dificuldade para a realização do exame. O instrumento apresentou validade aparente, validade de conteúdo, validade de construto, nível satisfatório de confiabilidade e validade discriminante. Conclusão: O instrumento de avaliação dos aspectos que influenciam a solicitação de exames na prática médica tem a possibilidade de identificar a influência particular de cada aspecto e de cada dimensão que interfere na prática de solicitação de exames.
\end{abstract}

Palavras-chave: testes diagnósticos de rotina; exames médicos; psicometria; estudos de validação; educação médica.

\footnotetext{
Abstract

Background: Exams are part of the health system, and they contribute to the advancement of care. The request of exams by physicians must be performed properly, and can be influenced by different aspects. Objective: Develop and validate an instrument to evaluate aspects that influence the request for exams. Method: This is a cross-sectional, analytical study conducted in the following steps: 1) Identification of the aspects that influence the request for exams in medical practice;2) Verification of apparent and content

${ }^{1}$ Universidade Estadual de Montes Claros (Unimontes) - Montes Claros (MG), Brasil.

${ }^{2}$ Faculdades Integradas Pitágoras - Montes Claros (MG), Brasil.

${ }^{3}$ Universidade do Estado de Minas Gerais (UEMG) - Passos (MG), Brasil.

Trabalho realizado pela Universidade Estadual de Montes Claros (Unimontes) - Montes Claros (MG), Brasil.

Endereço para correspondência: Maria Fernanda Santos Figueiredo Brito - Universidade Estadual de Montes Claros (Unimontes), Rua Borges Hermida, 346, ap. 103 - Major Prates - CEP: 39403-198 - Montes Claros (MG), Brasil - Email: mfsfbrito@yahoo.com.br

Fonte de financiamento: Fundação de Amparo à Pesquisa do Estado de Minas Gerais (FAPEMIG).

Conflito de interesses: nada a declarar.
}

\footnotetext{
* Manuscrito extraído da Tese de Doutorado de Maria Fernanda Santos Figueiredo Brito intitulada "Exames de apoio diagnóstico: a prática de médicos da Atenção Primária à Saúde e da Urgência e Emergência”, apresentada ao Programa de Pós Graduação em Ciências da Saúde da Universidade Estadual de Montes Claros no ano de 2014.
} 
validity; 3) Verification of construct validity; 4) Reliability analysis; 5) Verification of discriminant validity applied to 158 family and emergency physicians in the north of Minas Gerais state. Results: The instrument was composed of 36 psychometrically valid items grouped into five dimensions: particularity of the physician, structure and processes of the health system, characteristics of the patient, professional expertise, and difficulty conducting the exam. The instrument presented apparent and content validity, construct validity, satisfactory level of reliability, and discriminant validity. Conclusion: The instrument to assess aspects that influence the request of exams in medical practice can identify the particular influence of each aspect and each dimension that interferes with the practice of requesting exams.

Keywords: diagnostic tests; routine; medical examination; psychometrics; validation studies; education; medical.

\section{INTRODUÇÃO}

Os serviços de saúde enfrentam um importante desafio em sua organização, o qual envolve estrutura, processos e busca de resultados eficientes com o controle de custos em relação à carência de recursos ${ }^{1,2}$ e à expectativa do público ${ }^{3}$.

O uso excessivo de serviços médicos é um fator cada vez mais reconhecido de cuidados de baixa qualidade e alto custo, o que pode gerar decisões sobre cuidados inadequados em saúde ${ }^{4,5}$.

Os exames de apoio diagnóstico integram o sistema de saúde ${ }^{6}$ e contribuem para o avanço da assistência ${ }^{7}$, devendo ser indicados de forma apropriada pelos médicos ${ }^{78}$. Todavia, o uso excessivo desse recurso é uma realidade em muitos serviços, sendo motivo de preocupação atual, o que requer medidas de intervenção ${ }^{9}$.

Variações da prática médica são comuns ${ }^{10}$. A solicitação de exames na prática médica tem sido influenciada por vários aspectos, que contemplam as características do médico ${ }^{2,6,8,11}$, do paciente ${ }^{8,11}$, dos serviços de saúde ${ }^{11}$ e dos procedimentos diagnósticos ${ }^{6}$. Muitas vezes, ela é realizada de forma não sistematizada, até mesmo desnecessária ou inapropriada ${ }^{2,7,11}$, podendo resultar em iatrogenia ${ }^{7}$, em impactos financeiros negativos no sistema de saúde ${ }^{2}$ e no paciente ${ }^{7}$, em sobrecarga dos serviços propedêuticos, com repercussões na sustentabilidade do sistema de saúde ${ }^{8}$.

Ferramentas para aprimorar a solicitação de exames têm sido implementadas no cenário internacional, como os sistemas de suporte de decisão clínica ${ }^{11}$, os protocolos ${ }^{8}$ e a medicina baseada em evidências ${ }^{2}$. Contudo, tais instrumentos não consideram os aspectos que influenciam a prática de solicitação de exames, o que é primordial para o aprimoramento dessas atividades ${ }^{2}$.

A avaliação dos aspectos que influenciam o profissional médico durante a solicitação de exames no contexto dos serviços de saúde é imprescindível ${ }^{6}$. Todavia, não há acompanhamento, avaliação e controle de todos esses aspectos nem existência de um instrumento consensual que possibilite tal análise. Entender a rede de influência da prática de solicitação de exames diagnósticos é fundamental para o desenvolvimento de intervenções efetivas que possibilitem o uso adequado dos recursos ${ }^{3,5}$.

Assim sendo, há a necessidade de criação de um instrumento que avalie os aspectos que influenciam a solicitação de exames pelos médicos e possua qualidades psicométricas e aplicabilidade na prática. Este estudo propõe desenvolver e validar um instrumento de avaliação dos aspectos que influenciam a solicitação de exames de apoio diagnóstico pelos médicos.

\section{MÉTODO}

Trata-se de um estudo transversal e analítico. Esta pesquisa foi submetida à apreciação do Comitê de Ética em Pesquisa da Universidade Estadual de Montes Claros e foi aprovada segundo parecer consubstanciado $n^{\circ} 2.963 / 2011$.

O estudo foi realizado nas cidades de Montes Claros, Pirapora, Januária e Janaúba, municípios-polo da região norte de Minas Gerais, na Atenção Primária à Saúde, em equipes de saúde da família e nos serviços hospitalares de urgência e emergência, por constituírem as principais portas de entrada do usuário ao sistema público de saúde. A população-alvo foram os médicos de família e os médicos emergencistas atuantes nesse cenário há pelo menos seis meses.

O processo de desenvolvimento e da análise das propriedades psicométricas do instrumento seguiu cinco etapas: 1) identificação dos aspectos que influenciam a solicitação de exames na prática médica; 2) verificação da validade aparente e validade de conteúdo; 3) verificação da validade de construto; 4) análise da confiabilidade; 5) verificação da validade discriminante

\section{1)Identificação dos aspectos que influenciam a solicitação de exames na prática médica}

A identificação dos aspectos que influenciam a solicitação de exames na prática médica foi realizada por meio de revisão de literatura de artigos publicados até o ano de 2011, nas bases de dados Medline, SciELO; Lilacs e Pubmed. Para tanto, foram utilizados os descritores em ciências da saúde: "testes diagnóstico de rotina", "atenção primária à saúde" e "urgências". A síntese dos aspectos identificados na literatura resultou em 63 itens, tendo o cuidado para que cada um representasse apenas um pensamento, formando sentenças curtas, objetivas e claras. Em seguida, os itens identificados foram submetidos às etapas de validação aparente e de conteúdo, validade de construto, análise de confiabilidade e validade discriminante.

\section{2) Validade aparente e validade de conteúdo}

A validação aparente e a validação de conteúdo foram realizadas por sete juízes, que dominavam o conteúdo envolvido e possuíam experiência profissional em relação à temática 
investigada. Para a validade aparente, os especialistas (juízes) analisaram os 63 itens quanto à adequação da estrutura semântica, à facilidade de compreensão e clareza, ao formato dos itens e à categorização das respostas. Para a validade de conteúdo, foi avaliado se os itens do instrumento eram representativos de todas as facetas do construto a ser medido. Dessa forma, os especialistas opinaram no que se refere à necessidade de retirar, acrescentar ou modificar os itens propostos. Foi sugerido o acréscimo de seis itens: 1) a necessidade de realização de controle de cura; 2) a existência de um formulário com o nome de todos os exames disponíveis para o médico marcar o(s) desejado(s); 3) a dificuldade ou a ausência de deambulação pelo paciente; 4) o fato de o serviço de saúde ser público; 5) a necessidade de consentimento do paciente ou da família para a realização do exame; 6) o paciente ser usuário de substâncias ilícitas.

Foram realizadas as adequações sugeridas pelos especialistas, e o instrumento preliminar de avaliação dos aspectos que influenciam a solicitação de exames na prática médica ficou composto de 69 itens. Em seguida, foram organizados em itens de forma aleatória, construindo um instrumento para avaliar o quanto cada um deles influenciava a prática de solicitação de exames pelos médicos. As respostas estavam na escala de Likert com cinco opções: 1 = nunca; 2 = raramente; 3 = ocasionalmente; $4=$ frequentemente; $5=$ sempre. Cinquenta e três itens presentes na escala eram positivos, indicando a sua influência para a maior solicitação, e 16 itens eram negativos, indicando a sua influência para a menor solicitação de exames.

A versão preliminar do instrumento foi aplicada à população-alvo que aceitou participar da pesquisa, no período de janeiro de 2012 a abril de 2013. As informações sociodemográficas, de formação e de atuação do médico pesquisado foram obtidas por meio de um questionário.

\section{3) Validade de construto}

A validade de construto foi realizada por meio da análise fatorial exploratória. Precedendo a sua realização, os 16 itens negativos tiveram os valores das suas respostas invertidos na escala de Likert: $(1=5),(2=4),(3=3),(4=2),(5=1)$, a fim de que todos os itens se tornassem positivos.

Para identificar as dimensões finais do construto que iria compor o instrumento e os itens a elas associados, iniciou-se o seu processo com a realização da análise fatorial exploratória, utilizando o método de componentes principais com Rotação Varimax. Para avaliar a adequação dos dados à análise fatorial exploratória, foi utilizado o teste de Kaiser-Meyer-Olkim (KMO), para o qual se adotou valor superior a $0,5^{12}$. A presença de correlação entre as variáveis foi medida pelo teste de esfericidade de Bartlett, considerando-se significativo valor de p menor do que 0,05 . Utilizaram-se como critério inicial para a retenção das dimensões aquelas que apresentaram autovalores acima de $1 \mathrm{e}$, posteriormente, a observação do diagrama de declividade e da proporção da variância explicada, avaliando concomitantemente a presença de significação clínica. Realizou-se a inspeção das cargas fatoriais de cada fator, excluindo-se aqueles com carga fatorial inferior a $0,40^{12}$. Naqueles itens que apresentaram carga fatorial a partir de 0,40 em mais de uma dimensão, o critério de alocação do item foi a significação clínica.

Analisaram-se ainda as correlações entre os escores dos domínios, e, para tal, foi adotado o coeficiente de correlação de Spearman, considerando-se significativos os coeficientes com valor de $\mathrm{p} \leq 0,05$.

\section{4)Análise de confiabilidade}

A confiabilidade do instrumento e de cada dimensão foi avaliada por meio da análise da consistência interna e da análise de reprodutibilidade. Para análise da consistência interna do instrumento, verificou-se o coeficiente de alfa de Cronbach, cujos valores acima de 0,70 foram considerados confiáveis ${ }^{12}$. Realizou-se a análise da fidedignidade individual dos itens, a qual indicou aqueles que deveriam ser excluídos, com a finalidade de aumentar a confiabilidade do instrumento e das dimensões ${ }^{12}$.

Para testar a reprodutibilidade do instrumento, o questionário foi aplicado e reaplicado aleatoriamente a 25 médicos participantes do estudo, atendendo à recomendação mínima de $10 \%$ da amostra $^{13}$, após o período de 15 dias. Em seguida, realizou-se o teste de Kappa ponderado. A interpretação dos coeficientes de Kappa foi realizada a partir dos seguintes critérios ${ }^{14}$ : sem concordância $(<0)$, concordância pobre $(0 \mathrm{a} 0,19)$, concordância razoável $(0,20$ a 0,39$)$, concordância moderada $(0,40$ a 0,59$)$, concordância substancial $(0,60$ a 0,79$)$ e excelente concordância $(0,80$ a 1,00$)$.

\section{5) Validade discriminante}

Para a realização da validade discriminante, foi computado o escore das dimensões retidas, por meio da somatória dos valores atribuídos na escala de Likert pelos médicos pesquisados aos itens que integravam cada dimensão. Em seguida, realizou-se a transformação dos escores na escala de 1 a 100. Procedeu-se à comparação dos escores das dimensões identificadas entre subgrupos populacionais que hipoteticamente deveriam apresentar níveis diferentes de influência das dimensões, utilizando-se o teste de Mann-Whitney, com nível de significância igual a 0,05. Esses subgrupos foram definidos a partir de características de formação profissional (realização de residência médica) e ocupacionais (local de atuação e tempo de atuação no atual serviço de saúde).

Todas as análises estatísticas foram realizadas com auxílio do Software Predictive Analytics SoftWare (PASW) ${ }^{\circledR}$, versão 18.0 para Windows ${ }^{\circledR}$. 


\section{RESULTADOS}

Participaram do estudo 158 médicos atuantes em equipes de saúde da família ou no serviço hospitalar de urgência e emergência. Primeiramente, os 69 itens identificados e organizados no instrumento preliminar foram aplicados aos médicos participantes e submetidos à validade de construto. O teste de Kaiser-Meyer-Olkin (KMO) de 0,607 e o teste de esfericidade de Bartlett, apresentando $\mathrm{p}=0,000$, demonstraram uma adequação satisfatória dos dados para a realização da validade de construto. $O$ procedimento da análise fatorial exploratória resultou na eliminação de 33 itens, dos quais 29 por possuírem carga fatorial inferior a 0,40. A Tabela 1 apresenta as cargas fatoriais de cada item nas cinco dimensões.

$\mathrm{Na}$ avaliação da confiabilidade, por meio na análise de consistência interna, quatro itens foram retirados por aumentarem o valor de alfa de Cronbach, resultando em 36 itens distribuídos em cinco dimensões, que apresentaram significação clínica, e 43,9\% da variância explicada, obtendo-se a versão final do instrumento (Figura 1).

As características psicométricas do instrumento, resultantes da análise fatorial exploratória e da análise de confiabilidade, estão demonstradas na Tabela 2. Os autovalores das dimensões

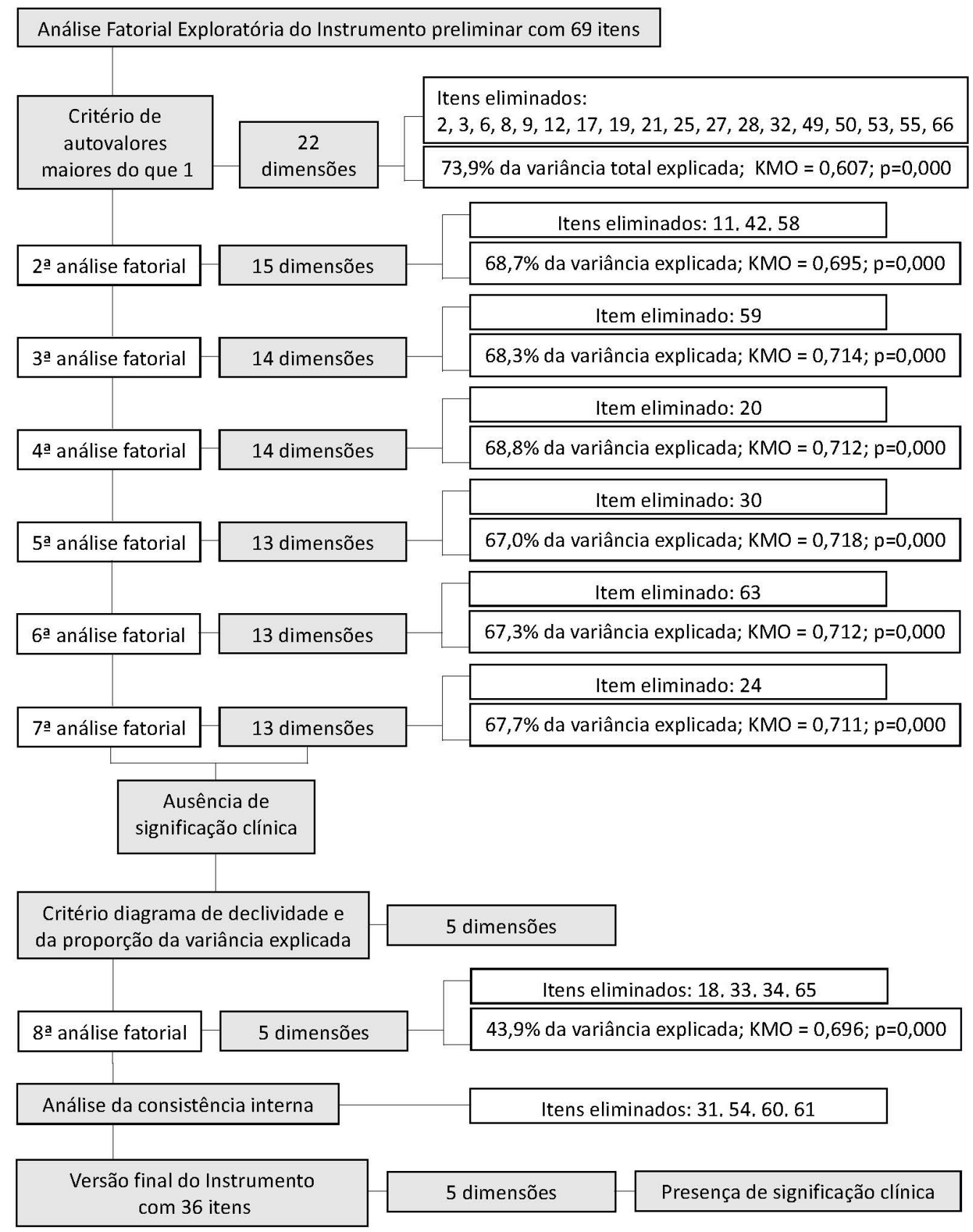

Figura 1. Etapas para a obtenção da versão final do Instrumento de Avaliação dos Aspectos que Influenciam a Solicitação de Exames , norte de Minas Gerais, Brasil, 2013 
Tabela 1. Cargas fatoriais dos 36 itens do Instrumento de Avaliação dos Aspectos que Influenciam a Solicitação de Exames por dimensões, norte de Minas Gerais, Brasil, 2013

\begin{tabular}{|c|c|c|c|c|c|c|}
\hline Item & Conteúdo & D1 & D2 & D3 & D4 & D5 \\
\hline 1 & $\begin{array}{l}\text { A gravidade do diagnóstico clínico do paciente te } \\
\text { influencia a solicitar mais os exames diagnósticos? }\end{array}$ & $-0,017$ & 0,018 & 0,400 & 0,056 & 0,032 \\
\hline 4 & $\begin{array}{l}\text { A gravidade de sintomas no paciente no momento } \\
\text { da consulta te influencia a solicitar mais os exames } \\
\text { diagnósticos? }\end{array}$ & 0,201 & $-0,169$ & 0,480 & $-0,053$ & $-0,095$ \\
\hline 5 & $\begin{array}{l}\text { O fato de o paciente apresentar mais de uma comorbidade } \\
\text { te influencia a solicitar mais os exames diagnósticos? }\end{array}$ & 0,122 & 0,059 & 0,744 & 0,108 & 0,062 \\
\hline 7 & $\begin{array}{l}\text { A presença de hábito tabagistas e etilistas do paciente te } \\
\text { influencia a solicitar mais os exames diagnósticos? }\end{array}$ & 0,322 & 0,151 & 0,469 & $-0,154$ & $-0,207$ \\
\hline 10 & $\begin{array}{l}\text { Gênero do paciente te influencia a solicitar mais os } \\
\text { exames diagnósticos? }\end{array}$ & 0,171 & 0,196 & 0,470 & 0,092 & $-0,074$ \\
\hline 13 & $\begin{array}{l}\text { De uma maneira geral, a presença de doença de } \\
\text { caráter familiar te influencia a solicitar mais os exames } \\
\text { diagnósticos? }\end{array}$ & 0,146 & 0,040 & 0,526 & $-0,273$ & 0,155 \\
\hline 14 & $\begin{array}{l}\text { A previsão para submissão a um procedimento } \\
\text { cirúrgico eletivo te influencia a solicitar mais os exames } \\
\text { diagnósticos? }\end{array}$ & $-0,144$ & 0,090 & 0,586 & 0,000 & 0,398 \\
\hline 15 & $\begin{array}{l}\text { A presença de fatores de risco no paciente (diabetes, } \\
\text { hipertensão etc.) te influencia a solicitar mais os exames } \\
\text { diagnósticos? }\end{array}$ & $-0,060$ & $-0,119$ & 0,518 & $-0,197$ & 0,481 \\
\hline 16 & $\begin{array}{l}\text { O fato de o paciente buscar frequentemente o } \\
\text { atendimento médico te influencia a solicitar mais os } \\
\text { exames diagnósticos? }\end{array}$ & 0,132 & 0,286 & 0,482 & 0,026 & $-0,027$ \\
\hline 22 & $\begin{array}{l}\text { A falta de resposta à terapêutica estabelecida te influencia } \\
\text { a solicitar mais os exames diagnósticos? }\end{array}$ & 0,431 & 0,022 & 0,404 & 0,048 & 0,149 \\
\hline 23 & $\begin{array}{l}\text { A demanda/pressão do paciente para solicitar } \\
\text { determinados exames diagnósticos te influencia a solicitá- } \\
\text { los mais? }\end{array}$ & 0,185 & 0,436 & 0,202 & 0,037 & 0,292 \\
\hline 24 & $\begin{array}{l}\text { A disponibilidade do exame diagnóstico no sistema } \\
\text { de saúde te influencia a solicitar mais os exames } \\
\text { diagnósticos? }\end{array}$ & 0,242 & 0,404 & 0,129 & $-0,066$ & 0,301 \\
\hline 26 & $\begin{array}{l}\text { O fato de existir formulários com o nome de todos os } \\
\text { exames disponíveis para o médico marcar os desejados te } \\
\text { influencia a solicitá-los mais? }\end{array}$ & 0,182 & 0,603 & 0,049 & 0,113 & $-0,104$ \\
\hline 29 & $\begin{array}{l}\text { O maior tempo de trabalho no serviço de saúde te } \\
\text { influencia a solicitar mais os exames diagnósticos? }\end{array}$ & 0,266 & 0,582 & 0,130 & 0,255 & $-0,129$ \\
\hline 35 & $\begin{array}{l}\text { A sua inexperiência em determinada situação clínica te } \\
\text { influencia a solicitar mais os exames diagnósticos? }\end{array}$ & 0,466 & 0,267 & 0,131 & $-0,156$ & 0,107 \\
\hline 36 & $\begin{array}{l}\text { O fato de um especialista sugerir determinado exame te } \\
\text { influencia a solicitá-lo? }\end{array}$ & 0,605 & 0,126 & $-0,081$ & 0,144 & 0,078 \\
\hline 37 & $\begin{array}{l}\text { O relacionamento médico-paciente deficiente te } \\
\text { influencia a solicitar mais os exames diagnósticos? }\end{array}$ & 0,270 & 0,588 & 0,196 & $-0,123$ & $-0,001$ \\
\hline 38 & $\begin{array}{l}\text { A dificuldade na tomada de decisão ou na condução do } \\
\text { caso do paciente te influencia a solicitar mais os exames } \\
\text { diagnósticos? }\end{array}$ & 0,527 & 0,337 & 0,222 & $-0,041$ & 0,000 \\
\hline 39 & $\begin{array}{l}\text { A segurança de que o exame diagnóstico realmente é } \\
\text { necessário na condução do caso do paciente te influencia } \\
\text { a solicitar mais o exame diagnóstico? }\end{array}$ & 0,483 & $-0,266$ & 0,010 & $-0,307$ & 0,245 \\
\hline 40 & $\begin{array}{l}\text { A necessidade de confirmação do diagnóstico por meio } \\
\text { de exames diagnósticos te influencia a solicitá-los mais? }\end{array}$ & 0,568 & $-0,225$ & 0,207 & $-0,246$ & 0,083 \\
\hline 41 & $\begin{array}{l}\text { A participação em cursos/congressos/capacitações sobre } \\
\text { determinada enfermidade te influencia a solicitar mais os } \\
\text { exames diagnósticos? }\end{array}$ & 0,467 & 0,071 & 0,109 & $-0,260$ & $-0,088$ \\
\hline
\end{tabular}

D1 = dimensão Particularidade pessoal do médico; D2 = dimensão Estrutura e processos do sistema de saúde; D3 = dimensão Características do paciente; D4 = dimensão Preparo profissional; D5 = dimensão Dificuldade para a realização do exame 
Tabela 1. Continuação...

\begin{tabular}{|c|c|c|c|c|c|c|}
\hline Item & Conteúdo & D1 & D2 & D3 & D4 & D5 \\
\hline 43 & $\begin{array}{l}\text { O fato de o exame diagnóstico apresentar altos índices } \\
\text { de sensibilidade, especificidade ou valor preditivo te } \\
\text { influencia a solicitar mais os exames diagnósticos? }\end{array}$ & 0,588 & $-0,097$ & 0,210 & $-0,328$ & 0,106 \\
\hline 44 & $\begin{array}{l}\text { O fato de o exame diagnóstico apresentar inovações } \\
\text { científicas e/ou tecnológicas te influencia a solicitá-lo } \\
\text { mais? }\end{array}$ & 0,471 & 0,089 & 0,186 & 0,108 & 0,004 \\
\hline 45 & $\begin{array}{l}\text { A relação custo-benefício favorável do exame diagnóstico } \\
\text { te influencia a solicitá-lo mais? }\end{array}$ & 0,423 & $-0,201$ & 0,015 & $-0,003$ & 0,507 \\
\hline 46 & $\begin{array}{l}\text { O receio de assumir risco do diagnóstico sem o respaldo } \\
\text { do exame diagnóstico te influencia a solicitar mais os } \\
\text { exames diagnósticos? }\end{array}$ & 0,659 & 0,176 & 0,016 & 0,085 & 0,181 \\
\hline 47 & $\begin{array}{l}\text { O receio de ações judiciais te influencia a solicitar mais os } \\
\text { exames diagnósticos? }\end{array}$ & 0,534 & 0,303 & 0,029 & 0,034 & $-0,051$ \\
\hline 48 & $\begin{array}{l}\text { O receio de arrepender-se posteriormente por não ter } \\
\text { pedido os exames diagnósticos te influencia a solicitá-los } \\
\text { mais? }\end{array}$ & 0,525 & 0,406 & 0,031 & 0,073 & $-0,154$ \\
\hline 51 & $\begin{array}{l}\text { A realização de marketing dos serviços propedêuticos } \\
\text { para a solicitação de determinados exames diagnósticos te } \\
\text { influencia a solicitá-los mais? }\end{array}$ & 0,059 & 0,605 & $-0,021$ & 0,225 & 0,065 \\
\hline 52 & $\begin{array}{l}\text { Se você tivesse um sistema de suporte de decisão clínica } \\
\text { que te fornecesse lembretes de indicação de exames } \\
\text { necessários, você solicitaria mais exames diagnósticos? }\end{array}$ & 0,467 & 0,142 & 0,036 & $-0,041$ & 0,364 \\
\hline 56 & $\begin{array}{l}\text { A realização de uma anamnese com informações } \\
\text { detalhadas te influencia a solicitar menos os exames } \\
\text { diagnósticos? }\end{array}$ & $-0,105$ & 0,206 & 0,003 & 0,738 & 0,022 \\
\hline 57 & $\begin{array}{l}\text { A realização de um exame clínico minucioso do paciente } \\
\text { te influencia a solicitar menos os exames diagnósticos? }\end{array}$ & $-0,103$ & 0,146 & $-0,011$ & 0,675 & 0,074 \\
\hline 62 & $\begin{array}{l}\text { Participação em atividades de educação permanente, com } \\
\text { discussão dos casos em pequenos grupos, te influencia a } \\
\text { solicitar menos os exames diagnósticos? }\end{array}$ & 0,108 & $-0,189$ & 0,091 & 0,715 & $-0,141$ \\
\hline 64 & $\begin{array}{l}\text { O fato de haver restrição para a realização do exame } \\
\text { diagnóstico pelo serviço de saúde (cotas, não oferecer/não } \\
\text { autorizar o exame) te influencia a solicitá-lo menos? }\end{array}$ & $-0,086$ & $-0,365$ & $-0,036$ & $-0,105$ & $-0,577$ \\
\hline 67 & $\begin{array}{l}\text { O conhecimento sobre os riscos inerentes ao exame } \\
\text { diagnóstico te influencia a solicitá-lo menos? }\end{array}$ & $-0,078$ & $-0,038$ & 0,073 & 0,346 & $-0,485$ \\
\hline 68 & $\begin{array}{l}\text { O conhecimento sobre o alto custo do exame diagnóstico } \\
\text { te influencia a solicitá-lo menos? }\end{array}$ & $-0,058$ & $-0,183$ & $-0,036$ & 0,055 & $-0,748$ \\
\hline 69 & $\begin{array}{l}\text { A certeza de que você terá outras oportunidades de } \\
\text { acompanhar o seu paciente te faz solicitar menos exames } \\
\text { diagnósticos? }\end{array}$ & 0,030 & $-0,201$ & $-0,030$ & 0,680 & $-0,102$ \\
\hline
\end{tabular}

D1 = dimensão Particularidade pessoal do médico; D2 = dimensão Estrutura e processos do sistema de saúde; D3 = dimensão Características do paciente; D4 = dimensão Preparo profissional; D5 = dimensão Dificuldade para a realização do exame

variaram de 2,64 a 4,58, com a dimensão 1 explicando 11,44\% da variância, e a dimensão 5, 6,61\%. O instrumento apresentou nível de consistência interna satisfatório, expresso pelo coeficiente alfa de Cronbach, com valor igual a 0,79. As dimensões de 1 a 4 também apresentaram níveis individuais adequados de consistência interna, e a dimensão 5, limítrofe. O teste de reprodutibilidade do instrumento apresentou valor de Kappa de 0,53 , indicando concordância moderada, o que também foi verificado nas cinco dimensões (Tabela 2 ).

Os itens $15,22,45$ e 48 tiveram cargas fatoriais a partir de 0,40 em duas dimensões (Tabela 1). O padrão de cargas cruzadas foi examinado minuciosamente. Nenhum desses itens foi eliminado por essa situação, pois era imprescindível ao conteúdo do instrumento. A alocação desses itens em uma das dimensões foi estabelecida pela coerência e significação clínica.

A dimensão 1 foi denominada "Particularidade pessoal do médico" e se refere às características comportamentais peculiares de cada profissional médico, de acordo com a disposição da sua personalidade e da sua sensibilidade, que faz com que ele perceba, de modo particular, a influência, em sua conduta profissional, das suas próprias características, das características do exame, da sugestão de especialistas, de cursos e de suportes tecnológicos. Ela foi composta por 13 itens $(35,36,38,39$, $40,41,43,44,45,46,47,48,52$ ). A dimensão 2 foi intitulada 
"Estrutura e processos do sistema de saúde" e diz respeito aos aspectos não relacionados às características do médico e do paciente. Refere-se à estrutura e aos processos locais do sistema de saúde. Ela foi representada por 6 itens $(23,24,26,29,37,51)$. A dimensão 3 foi nomeada “Características do paciente" e se relaciona aos aspectos sociodemográficos e clínicos dos pacientes. Ela englobou 10 itens $(1,4,5,7,10,13,14,15,16,22)$. A dimensão 4 foi designada “Preparo profissional” e faz referência ao preparo e à segurança do médico para a realização de um atendimento clínico de qualidade e a sua participação em processos de capacitação profissional. Ela foi formada por 4 itens $(56,57,62,69)$. A dimensão 5 foi denominada "Dificuldade para a realização do exame" e compreende os aspectos dificultadores para a realização do exame. Ela envolveu 3 itens $(64,67,68)$.

Quanto às correlações entre os domínios, os valores dos coeficientes de correlação de Spearman variaram de 0,02 (entre as dimensões "Estrutura e processos do sistema de saúde" e "Preparo profissional") a 0,45 (entre as dimensões "Particularidade pessoal do médico" e "Estrutura e processos do sistema de saúde"). A maioria desses coeficientes foi significativa ( $p<0,05)$, com exceção daqueles que mediram as correlações entre a dimensão "Preparo profissional” e as dimensões "Particularidade pessoal do médico", "Estrutura e processos do sistema de saúde" e "Características do paciente".

A validade discriminante foi verificada no instrumento proposto, uma vez que os subgrupos populacionais investigados - realização de residência médica, local de atuação e tempo de atuação no atual serviço de saúde - apresentaram distinção quanto à influência das cinco dimensões que envolvem a prática de solicitação de exames pelo médico. Os escores da dimensão "Dificuldade para a realização do exame", segundo as características de formação profissional e ocupação, apresentaram diferenças estatisticamente significantes. A dimensão "Particularidade pessoal do médico" teve diferença estatisticamente significante entre os médicos que trabalham na Estratégia Saúde da Família ou na urgência e emergência e entre os que trabalham há dez anos ou mais no atual serviço de saúde ou há menos de dez anos, e limítrofe entre os que possuem ou não residência médica. A dimensão "Estrutura e processos do sistema de saúde" apresentou associação estatisticamente significante entre os indivíduos que possuem ou não residência médica, e a dimensão "Características do paciente", entre os indivíduos que trabalham na Estratégia Saúde da Família ou na urgência e emergência. A dimensão "Preparo profissional" não teve diferença estatisticamente significante apenas entre os indivíduos que possuem ou não residência médica (Tabela 3 ).

Tabela 2. Características psicométricas do Instrumento de Avaliação dos Aspectos que Influenciam a Solicitação de Exames, norte de Minas Gerais, Brasil, 2013

\begin{tabular}{|c|c|c|c|c|c|}
\hline Características psicométricas & D1 & D2 & D3 & D4 & D5 \\
\hline Autovalor & 4,58 & 4,39 & 3,07 & 2,88 & 2,64 \\
\hline Porcentagem da variância explicada & $11,44 \%$ & $10,97 \%$ & $7,67 \%$ & $7,21 \%$ & $6,61 \%$ \\
\hline $\begin{array}{l}\text { Consistência interna } \\
\text { (alfa de Cronbach) }\end{array}$ & 0,81 & 0,72 & 0,73 & 0,79 & 0,65 \\
\hline Reprodutibilidade (Kappa) & 0,52 & 0,55 & 0,53 & 0,45 & 0,56 \\
\hline
\end{tabular}

D1 = dimensão Particularidade pessoal do médico; D2 = dimensão Estrutura e processos do sistema de saúde; D3 = dimensão Características do paciente; D4 = dimensão Preparo profissional; D5 = dimensão Dificuldade para a realização do exame

Tabela 3. Validade discriminante do Instrumento de Avaliação dos Aspectos que Influenciam a Solicitação de Exames, norte de Minas Gerais, Brasil, 2013

\begin{tabular}{|c|c|c|c|c|c|c|c|c|c|c|c|c|}
\hline \multirow{2}{*}{ Características } & \multirow{2}{*}{$\mathbf{N}$} & \multirow{2}{*}{$\%$} & \multicolumn{2}{|c|}{ D1 } & \multicolumn{2}{|c|}{ D2 } & \multicolumn{2}{|c|}{ D3 } & \multicolumn{2}{|c|}{ D4 } & \multicolumn{2}{|c|}{ D5 } \\
\hline & & & Mediana & $\overline{p \text {-valor }}$ & Mediana & $\overline{\text { p-valor }}$ & Mediana & $\overline{p \text {-valor }}$ & Mediana & p-valor & Mediana & p-valor \\
\hline \multicolumn{13}{|c|}{ Aspectos relacionados à formação médica } \\
\hline \multicolumn{13}{|c|}{ Residência médica } \\
\hline Sim & 55 & 34,8 & 59,0 & 0,058 & 28,9 & 0,015 & 59,4 & 0,162 & 68,1 & 0,294 & 57,8 & 0,034 \\
\hline Não & 103 & 65,2 & 55,2 & & 20,6 & & 54,5 & & 68,1 & & 49,5 & \\
\hline \multicolumn{13}{|l|}{ Aspectos ocupacionais } \\
\hline \multicolumn{13}{|c|}{ Setor de atenção à saúde } \\
\hline ESF & 70 & 44,3 & 60,9 & 0,044 & 24,8 & 0,137 & 56,9 & 0,018 & 71,2 & 0,018 & 57,8 & 0,000 \\
\hline Urgência e Emergência & 88 & 55,7 & 55,2 & & 24,8 & & 55,7 & & 61,9 & & 41,3 & \\
\hline \multicolumn{13}{|c|}{ Tempo que trabalha no atual serviço } \\
\hline Dez anos ou mais & 19 & 12,0 & 49,5 & 0,037 & 16,5 & 0,076 & 54,5 & 0,090 & 55,7 & 0,005 & 33,0 & 0,002 \\
\hline Menos de dez anos & 139 & 88,0 & 59,0 & & 24,8 & & 56,9 & & 68,1 & & 57,8 & \\
\hline
\end{tabular}

D1 = dimensão Particularidade pessoal do médico; D2 = dimensão Estrutura e processos do sistema de saúde; D3 = dimensão Características do paciente; D4 = dimensão Preparo profissional; D5 = dimensão Dificuldade para a realização do exame 


\section{DISCUSSÃO}

Este estudo desenvolveu e validou um instrumento de avaliação dos aspectos que influenciam a prática de solicitação de exames de apoio diagnóstico pelos médicos, composto por 36 itens, distribuídos em cinco dimensões. Os estudos presentes na literatura abordavam os fatores que influenciam, de modo geral, o uso excessivo dos serviços de saúde ${ }^{4,10}$ ou identificavam os aspectos influenciadores da solicitação de exames de forma não sistematizada ${ }^{6,8,11}$, não havendo instrumentos específicos para essa finalidade e avaliados quanto às suas qualidades psicométricas.

Na prática clínica, o uso de instrumentos qualificados direciona as investigações com maior segurança e fornece aos pesquisadores novos recursos metodológicos, sobretudo ao considerar a escassez de medidas validadas e normatizadas ${ }^{15}$. Contudo, há ainda uma carência desses instrumentos ${ }^{16}$, principalmente para avaliação de fenômenos subjetivos ${ }^{17}$.

O instrumento desenvolvido demonstrou ser válido, atendendo aos critérios de validade aparente, validade de conteúdo, validade de construto, confiabilidade e validade discriminante. Os itens do instrumento foram compreensíveis aos médicos, representando adequadamente as facetas importantes do construto estudado. Essa etapa deve ser considerada por pesquisadores e profissionais de saúde preocupados em utilizar instrumentos confiáveis e adequados à população de estudo ${ }^{18}$.

O processo de validade de construto realizado neste estudo possibilitou verificar a legitimidade da representação comportamental dos traços latentes ${ }^{19}$ e demonstrou que os itens identificados correspondem àqueles descritos pela teoria ${ }^{20}$. Quanto à adequação do número de sujeitos investigados para realização da análise fatorial exploratória, este estudo superou a recomendação mínima de 100 participantes ${ }^{21}$ e demonstrou adequabilidade da amostra para a realização da análise fatorial e fatorabilidade da matriz de correlações, sem prejuízos para essa validade.

A redução do número de itens durante essa etapa, em aproximadamente duas vezes, possibilitou que a versão final do instrumento apresentasse aplicabilidade prática, com menor tempo despendido para a sua aplicação, fator que deve ser considerado ao se desenvolver um instrumento, principalmente, aos que são voltados para os profissionais de saúde, tendo em vista a dinamicidade dos serviços de saúde e a carga de trabalho desses profissionais ${ }^{1}$.

A análise fatorial revelou que o instrumento pode ser interpretado como multidimensional. Entretanto, a retenção inicial de 13 dimensões indicadas pelo critério do autovalor (acima de 1) não apresentou significância clínica e ordem prática de análise, o que foi obtida por meio da solução de cinco dimensões evidenciadas pelo diagrama de declividade e da proporção da variância explicada. O critério de retenção de dimensões possibilitou considerar não apenas os parâmetros estatísticos, mas também o princípio de que os itens devem ser compreensíveis, teoricamente justificáveis e coerentes com a realidade prática. Por meio da análise fatorial, foi possível verificar que as dimensões subjacentes são as causas do construto estudado $^{20}$. A identificação de dimensões que influenciam a solicitação de exames, no presente trabalho, pode direcionar as organizações a implementar intervenções por domínios, o que facilita a sua aplicação ${ }^{4}$.

As cinco dimensões identificadas mostraram que os aspectos que influenciam a solicitação de exames podem ser agrupados diferentemente das formas apresentadas pela literatura internacional ${ }^{2,6,11}$. Os estudos, geralmente, classificam os itens que influenciam a solicitação de exames em aspectos relacionados ao médico ${ }^{2,6}$ e ao paciente ${ }^{2}$. Outra possibilidade de agrupamento desses aspectos sugerida em um trabalho desenvolvido nos Estados Unidos, além dos dois já citados, seria aspectos relacionados ao serviço e à comunidade. Porém, os autores limitaram a pesquisar os aspectos relacionados ao paciente e ao médico ${ }^{2}$. Estudo realizado no Canadá mostrou uma diferente maneira de ordenar esses aspectos - razões não clínicas e comunicação entre os médicos de diferentes especialidades ${ }^{11}$. Já outros estudos ${ }^{7,8}$ apontam alguns aspectos que influenciam a solicitação de exames pelos médicos sem sugerir o seu agrupamento. Ressalta-se que nenhum dos estudos $\operatorname{citados}^{2,6,11}$, que realizaram o agrupamento dos aspectos que influenciam a prática de solicitação pelos médicos, fundamentou-se em técnica estatística, especialmente na análise fatorial exploratória, que é a técnica apropriada para esse fim ${ }^{12}$.

O presente trabalho apontou que os aspectos relacionados ao médico podem ser analisados de forma compartimentalizada - "Particularidade pessoal do médico" e "Preparo profissional", diferentemente do estudo realizado nos Estados Unidos, que não fez tal distinção ${ }^{2}$. Essa subdivisão possibilita identificar, de forma distinta, a influência desses dois aspectos na prática de solicitação de exames, de modo que os esforços para o aprimoramento da prática profissional possam ser direcionados especificamente para áreas de necessidade de aprendizagem do profissional. A opção de não inserir os aspectos sociodemográficos do médico no presente instrumento, apesar de sua influência ter sido identificada em estudos internacionais ${ }^{2,6,11}$, deve-se ao fato de corresponderem a variáveis não modificáveis ${ }^{6}$. O reconhecimento das variáveis que são modificáveis tem sido apontado como essencial para aperfeiçoar a prática de solicitação de exames, tendo em vista a possibilidade de estabelecer intervenções que consideram essas variáveis ${ }^{6}$.

A influência das características sociodemográficas, culturais e clínicas dos pacientes, como mostrado neste trabalho, pode ser analisada conjuntamente, o que está em concordância com estudo desenvolvido no cenário internacional ${ }^{2}$. O desenvolvimento de uma estrutura que avalia especificamente os aspectos relacionados 
ao paciente tem sido ressaltado na literatura. Uma abordagem centrada no paciente otimiza os resultados para o indivíduo ${ }^{4}$.

No presente trabalho, verificou-se que alguns itens foram alocados em suas dimensões diferentemente do encontrado em outros trabalhos ${ }^{2,11}$. Tal fato era de se esperar devido às diferenças no número e nas formas de agrupamento dos itens entre os estudos internacionais e o presente estudo.

$\mathrm{O}$ instrumento desenvolvido apresentou consistência interna satisfatória e mostrou-se confiável, com itens altamente intercorrelacionados na medida do mesmo construto latente ${ }^{21}$.

A reprodutividade moderada observada pode estar relacionada ao fato de os aspectos que influenciam a prática de solicitação de exames não serem tema comum de estudo no meio médico ${ }^{11}$. A aplicação do instrumento aqui validado pode ser utilizada para diagnóstico e constituir uma oportunidade para reflexão, estudo e posterior mudança de atitude do médico.

Outro aspecto que precisa ser considerado é a interferência na reprodutibilidade do instrumento de aspectos pessoais transitórios, como fadiga, fome, ansiedade e estado de ânimo, e aspectos ambientais aos quais os participantes estão expostos ${ }^{22}$, pois, no caso do profissional médico, pode estar aumentada devido à dinamicidade dos serviços de saúde e à sobrecarga de trabalho desses profissionais ${ }^{1}$.

A existência de validade discriminante é importante, pois pode fornecer parâmetros para a comparação com os escores sobre os aspectos que influenciam a solicitação de exames pelos médicos em diferentes pontos de atenção em saúde. Tal fato ilustra como as diferentes dimensões da solicitação de exames podem também estar associadas à inserção e especialidade do profissional e à estrutura do serviço em si.

Esse instrumento tem utilidade para a autoavaliação do profissional médico sobre sua prática de solicitação de exames e para a análise da causa raiz de problemas relacionados à indicação inadequada dos recursos propedêuticos, contribuindo para a implementação de esforços em áreas prioritárias com o objetivo de reduzir o uso inapropriado direcionado a domínios específicos ${ }^{4}$. A redução da utilização inadequada dos recursos propedêuticos de saúde pode contribuir para melhorar a assistência de saúde à população, melhorar a experiência do cuidado e reduzir os gastos dos serviços de saúde ${ }^{5}$.

Constitui uma limitação deste estudo o fato de a estrutura dimensional não ter sido submetida a um processo de modelagem do tipo confirmatório, obtida por meio da análise fatorial confirmatória. No entanto, há a recomendação de que esse tipo de análise seja, preferencialmente, realizado em novo conjunto de dados ${ }^{23}$.

$\mathrm{O}$ instrumento de avaliação dos aspectos que influenciam a solicitação de exames na prática médica apresentou validade aparente, validade de conteúdo, validade de construto, nível satisfatório de confiabilidade e validade discriminante, sendo de fácil aplicação. Tem a possibilidade de identificar a influência particular de cada item e de cada dimensão que interfere na prática de solicitação de exames.

Espera-se que o uso do instrumento apresentado neste estudo possa subsidiar melhoria na preparação e mudança de atitude do profissional médico, na estrutura e organização dos serviços propedêuticos de saúde, na educação em saúde para os pacientes e na ergonomia dos equipamentos, processos e procedimentos realizados. É desejável a validação desse instrumento em amostras com outros níveis de atenção a fim de se refinar o instrumento. Sugere-se que futuros estudos sejam desenvolvidos com o uso de pesos diferenciados para os exames laboratoriais e de imagem.

\section{REFERÊNCIAS}

1. Tanaka OY, Tamaki EM. O papel da avaliação para a tomada de decisão na gestão de serviços de saúde. Cien Saude Colet. 2012;17(4):821-8. http:// dx.doi.org/10.1590/S1413-81232012000400002. PMid:22534834.

2. Sistrom C, McKay NL, Weilburg JB, Atlas SJ, Ferris TG. Determinants of diagnostic imaging utilization in primary care. Am J Manag Care. 2012;18(4):e135-44. PMid:22554039.

3. Goodman DC, Goodman AA. Medical care epidemiology and unwarranted variation: the Israeli case. Isr J Health Policy Res. 2017;6(9):1. http://dx.doi. org/10.1186/s13584-017-0135-6. PMid:28239448.

4. Morgan DJ, Leppin AL, Smith CD, Korenstein D. A practical framework for understanding and reducing medical overuse: conceptualizing overuse through the patient-clinician interaction. J Hosp Med. 2017;12(5):346-51. http://dx.doi.org/10.12788/jhm.2738. PMid:28459906.

5. Nassery N, Segal JB, Chang E, Bridges JF. Systematic overuse of healthcare services: a conceptual model. Appl Health Econ Health Policy. 2015;13(1):16. http://dx.doi.org/10.1007/s40258-014-0126-5. PMid:25193241.

6. Sood R, Sood A, Ghosh AK. Non-evidence-based variables affecting physicians' test-ordering tendencies: a systematic review. Neth J Med. 2007;65(5):167-77. PMid:17519512.

7. Aguiar FJB, Ferreira-Júnior M, Sales MM, Cruz-Neto LM, Fonseca LAM, Sumita NM, et al. C-reactive protein: clinical applications and proposals for a rational use. Rev Assoc Med Bras. 2013;59(1):85-92. http://dx.doi. org/10.1590/S0104-42302013000100016. PMid:23440147.

8. Morrison A. Appropriate utilization of advanced diagnostic imaging procedures: CT, MRI, and PET/CT [Internet]. Ottawa: Canadian Agency for Drugs and Technologies in Health. (Environmental Scan; 39) [citado em 2018 jul 5]. Disponível em: http://www.cadth.ca/media/pdf/ PFDIESLiteratureScan_e_es.pdf

9. Chan KS, Chang E, Nassery N, Chang HY, Segal JB. The state of overuse measurement: a critical review. Med Care Res Rev. 2013;70(5):473-96. http://dx.doi.org/10.1177/1077558713492202. PMid:23804290.

10. Wennberg JE. Tracking medicine: a researcher's quest to understand health care. New York: Oxford University Press; 2013. 
11. You JJ, Levinson W, Laupacis A. Attitudes of family physicians, specialists and radiologists about the use of computed tomography and magnetic resonance imaging in ontario. Healthc Policy. 2009;5(1):54-65. PMid:20676251.

12. Field A. Descobrindo a estatística usando o SPSS. 2. ed. Porto Alegre: Artmed; 2009.

13. Brasil. Ministério da Saúde. Secretaria de Políticas de Saúde. Departamento de Atenção Básica. Área Técnica de Saúde Bucal. Projeto SB2000: condições de saúde bucal da população brasileira no ano 2000: manual de calibração de examinadores. Brasília: Ministério da Saúde; 2001.

14. Landis JR, Koch GG. The measurement of observer agreement for categorical data. Biometrics. 1977;33(1):159-74. http://dx.doi.org/10.2307/2529310. PMid:843571.

15. Tozzi RC, Gonçalves GL, Simon HC. O processo de construção de escalas psicométricas. Aval Psicol. 2014;13(2):307-10.

16. Pawlowski J, Trentini CM, Bandeira DR. Discutindo procedimentos psicométricos a partir da análise de um instrumento de avaliação neuropsicológica breve. Psico-USF. 2007;12(2):211-9. http://dx.doi. org/10.1590/S1413-82712007000200009.

17. Mota DDC, Pimenta CAM. Avaliação e mensuração de variáveis psicossociais: desafio para pesquisa e clínica de enfermagem. Rev Gaúcha Enferm. 2007;28(3):309-14. PMid:18183691.
18. Alexandre NMC, Coluci MZO. Validade de conteúdo nos processos de construção e adaptação de instrumentos de medidas. Ciênc. Saúde Coletiva. 2011;16(7):3061-8. http://dx.doi.org/10.1590/S1413-81232011000800006.

19. Pasquali L. Psychometrics. Rev Esc Enferm USP. 2009;43(spe):992-9. http:// dx.doi.org/10.1590/S0080-62342009000500002.

20. Hutz CS, Bandeira DR, Trentini CM. Psicometria [Internet]. Porto Alegre: Artmed; 2015 [citado em 2018 jul 5]. Disponível em: https://pt.scribd.com/ document/373749525/Psicometria-Hutz-Bandeira-Trentini

21. Hair JF, Black WC, Babin BJ, Anderson RE, Tatham RL. Análise multivariada de dados. Porto Alegre: Bookman; 2009.

22. Perroca MG, Gaidzinski RR. Avaliando a confiabilidade interavaliadores de um instrumento para classificação de pacientes: coeficiente Kappa. Rev Esc Enferm USP. 2003;37(1):72-80. http://dx.doi.org/10.1590/S008062342003000100009 .

23. Hancock G, Mueller R, Stapleton L. The reviewer's guide to quantitative methods in the social sciences [Internet]. New York: Routledge; 2003 [citado em 2018 jun 10]. Disponível em: https://www.taylorfrancis.com/ books/9781135172992

Recebido em: Jan. 16, 2017 Aprovado em: Jul. 18, 2018 